\title{
Influence of Quasiperiodic Gravitational Modulation on Convective Instability of Liquid-Liquid Polymerization Front
}

\author{
Saadia Assiyad, ${ }^{1}$ Karam Allali, ${ }^{1}$ and Mohamed Belhaq ${ }^{2}$ \\ ${ }^{1}$ Department of Mathematics, University of Hassan II-Casablanca, FST, P.O. Box 146, Mohammedia, Morocco \\ ${ }^{2}$ Department of Mechanics, University of Hassan II-Casablanca, Casablanca, Morocco \\ Correspondence should be addressed to Karam Allali; allali@hotmail.com
}

Received 11 August 2015; Accepted 7 October 2015

Academic Editor: Fazal M. Mahomed

Copyright (c) 2015 Saadia Assiyad et al. This is an open access article distributed under the Creative Commons Attribution License, which permits unrestricted use, distribution, and reproduction in any medium, provided the original work is properly cited.

\begin{abstract}
The influence of quasiperiodic gravitational modulation on convective instability of polymerization front with liquid monomer and liquid polymer is studied. The model includes the heat equation, the concentration equation, and the Navier-Stokes equations under the Boussinesq approximation. The linear stability analysis of the problem is carried out and the interface problem is derived. Using numerical simulations, the convective instability threshold is determined and the boundary of the convective instability is obtained for different amplitudes and frequencies ratio.
\end{abstract}

\section{Introduction}

Frontal polymerization is the process of polymer production in propagating reaction fronts [1-4]. In the absence of vibration, the influence of convective instability on polymerization front when the monomer is liquid and the polymer is solid was studied in [5], while the case of liquid polymer was considered in [6]. The influence of periodic gravitational modulation on the convective instability in the case of liquidsolid polymerization front was studied [7] and it was shown that the propagation of polymerization reaction front is strongly affected by the amplitude and the frequency of vibrations. In particular, the polymerization front can be stable or unstable depending on the values of vibration parameters. The influence of periodic vibrations on convective instability of reaction front was also studied in the case of liquids [8] and it was concluded that, for small vibration amplitudes, the reaction front remains stable and it loses its stability for sufficiently large amplitude of vibrations.

Recent works were devoted to the influence of a quasiperiodic (QP) gravitational modulation on the convective instability of reaction front. For instance, the influence of the QP gravitational modulation on reaction front was examined in the case of porous media described by the Darcy equation [9]. On the other hand, the case of liquid-solid polymerization front was considered in [10] using the NavierStokes equations instead of the Darcy equation and it was revealed that both the amplitudes and the frequencies ratio influence the stability domain of the polymerization front. Specifically, for appropriate values of vibration amplitudes and increasing values of the frequencies ratio, a stabilizing effect is observed. The effect of the wave number on the reaction front was also examined showing that increasing the wave number widens the stability domain.

The present work studies the effect of QP gravitational modulation on the convective instability of the polymerization front, but in the case of liquid-liquid frontal polymerization. This case is different from the previous one [10] in the sense that in [10] the equation of motion is considered only after the reaction zone since the polymer is in the solid phase. In the present work instead, the equation of motion is considered before and after the reaction zone because both the monomer and the polymer are liquids.

The next section presents the frontal polymerization model. Section 3 develops the perturbation analysis, while the interface problem is examined in Section 4. The linear stability analysis is discussed in Section 5 and numerical investigations are carried out in Section 6. The last section concludes the work. 


\section{Frontal Polymerization Model}

We consider a polymerization process with a liquid reactant and a liquid product by assuming that the reaction front propagates in the direction opposite to the direction of gravity. In this case, the model of the frontal polymerization is given by the system of equations:

$$
\begin{aligned}
\frac{\partial T}{\partial t}+(v \cdot \nabla) T= & \kappa \Delta T+q W, \\
\frac{\partial \alpha}{\partial t}+(v \cdot \nabla) \alpha= & W, \\
\frac{\partial v}{\partial t}+(v \cdot \nabla) v= & -\frac{1}{\rho} \nabla p+v \Delta v \\
& +g(1+b(t)) \beta\left(T-T_{0}\right) \gamma, \\
\operatorname{div}(v)= & 0,
\end{aligned}
$$

with the following boundary conditions:

$$
\begin{aligned}
& z \longrightarrow+\infty: \quad T=T_{i}, \alpha=0, v=0, \\
& z \longrightarrow-\infty: \quad T=T_{b}, \alpha=1, v=0 .
\end{aligned}
$$

The gradient, divergence, and Laplace operators are defined by

$$
\begin{aligned}
\nabla v & =\left(\frac{\partial v}{\partial x}, \frac{\partial v}{\partial y}, \frac{\partial v}{\partial z}\right) \\
\operatorname{div} \overrightarrow{\mathbf{v}} & =\frac{\partial \mathbf{v}_{1}}{\partial x}+\frac{\partial \mathbf{v}_{2}}{\partial y}+\frac{\partial \mathbf{v}_{3}}{\partial z} \\
\Delta v & =\frac{\partial^{2} v}{\partial^{2} x}+\frac{\partial^{2} v}{\partial^{2} y}+\frac{\partial^{2} v}{\partial^{2} z}
\end{aligned}
$$

where the variables $(x, y$, and $z)$ are the spatial coordinates such that $-\infty<x, y, z<+\infty, T$ is the temperature, $\alpha$ is the concentration of the reaction product, $v$ is the velocity, $p$ is the pressure, $\kappa$ is the coefficient of thermal diffusivity, $q$ is the adiabatic temperature heat release, $\rho$ is an average value of the density, $\nu$ is the coefficient of kinematic viscosity, $\gamma$ is the unit vector in the upward direction, $\beta$ is the coefficient of thermal expansion, $g$ is the gravitational acceleration, and $b(t)$ is the QP acceleration acting on the fluid which is given by $b(t)=$ $\lambda_{1} \sin \left(\sigma_{1} t\right)+\lambda_{2} \sin \left(\sigma_{2} t\right)$ such that $\lambda_{1}, \lambda_{2}$ are the amplitudes and $\sigma_{1}, \sigma_{2}$ are the incommensurate frequencies of the QP gravitational modulation. The quantity $T_{0}$ is a mean value of temperature, $T_{i}$ is the initial temperature, and $T_{b}=T_{i}+q$ is the temperature of the reacted mixture. We consider onestep reaction of zero order where the reaction rate is defined as follows:

$$
W=k(T) \phi(\alpha), \quad \phi(\alpha)= \begin{cases}1 & \text { if } \alpha<1 \\ 0 & \text { if } \alpha=1\end{cases}
$$

The temperature dependence of the reaction rate is given by the Arrhenius law [11]

$$
k(T)=k_{0} \exp \left(-\frac{E}{R_{0} T}\right),
$$

where $k_{0}$ is the preexponential factor, $E$ is the activation energy, and $R_{0}$ is the universal gas constant. We assume that the two liquids are incompressible and the diffusivity coefficient is very small comparing to the thermal diffusivity coefficient such that the diffusivity will be neglected in the concentration equation.

To obtain the dimensionless model, we introduce the dimensionless spatial variables as

$$
\begin{aligned}
& x_{1}=\frac{x c_{1}}{\kappa}, \\
& y_{1}=\frac{y c_{1}}{\kappa} \\
& z_{1}=\frac{z c_{1}}{\kappa} \\
& t_{1}=\frac{t c_{1}^{2}}{\kappa} \\
& p_{1}=\frac{p}{c_{1}^{2} \rho}, \\
& c_{1}=\frac{c}{\sqrt{2}}, \\
& v_{1}=\frac{v}{c_{1}}, \\
& \theta=\frac{T-T_{b}}{q}, \\
& c^{2}=\frac{2 k_{0} \kappa R_{0} T_{b}^{2}}{q E} \exp \left(-\frac{E}{R_{0} T_{b}}\right),
\end{aligned}
$$

where $c$ defines the stationary reaction front velocity and can be calculated asymptotically for large Zeldovich number [12]. For simplicity, we keep the same notation for the other variables and pressure. System (1) with the two boundary conditions (2)-(3) can be written in the form

$$
\begin{aligned}
& \frac{\partial \theta}{\partial t}+(v \cdot \nabla) \theta=\Delta \theta+Z \exp \left(\frac{\theta}{Z^{-1}+\delta \theta}\right) \phi(\alpha), \\
& \frac{\partial \alpha}{\partial t}+(v \cdot \nabla) \alpha=Z \exp \left(\frac{\theta}{Z^{-1}+\delta \theta}\right) \phi(\alpha), \\
& \frac{\partial v}{\partial t}+(v \cdot \nabla) v \\
& \quad+\nabla p+P \Delta v \\
& \quad+P R\left(1+\lambda_{1} \sin \left(\sigma_{1} t\right)+\lambda_{2} \sin \left(\sigma_{2} t\right)\right)\left(\theta+\theta_{0}\right) \gamma, \\
& \operatorname{div}(v)=0,
\end{aligned}
$$

with the following conditions at infinity:

$$
\begin{aligned}
& z \longrightarrow+\infty: \quad \theta=-1, \alpha=0, v=0, \\
& z \longrightarrow-\infty: \quad \theta=0, \alpha=1, v=0
\end{aligned}
$$

and $P=\nu / \kappa$ is the Prandtl number, $R=g \beta q \kappa^{2} /\left(\nu c^{3}\right)$ is the Rayleigh number, $Z=q E / R_{0} T_{b}^{2}$ is the Zeldovich number, and 
$\delta$ and $\theta_{0}$ are given, respectively, by $\delta=R_{0} T_{b} / E$ and $\theta_{0}=\left(T_{b}-\right.$ $\left.T_{0}\right) / q$ and $\mu=2 \kappa \sigma / c^{2}$.

Next, we perform the linear stability analysis to tackle the interface problem.

\section{Approximation of Infinitely Narrow Reaction Zone}

To study the interface problem analytically, we use a singular perturbation analysis where the reaction zone is infinitely narrow and the reaction term is neglected outside the zone [13]. In this way, the problem can be reduced to an interface problem.

To perform a formal asymptotic analysis, $\epsilon=Z^{-1}$ is considered as a small parameter. The new independent variable is given by $z_{1}=z-\zeta(x, y, t)$ where $\zeta(x, y, t)$ defines the location of the reaction zone. Introducing new functions $\theta_{1}$, $\alpha_{1}, v_{1}$, and $p_{1}$ as

$$
\begin{aligned}
& \theta(x, y, z, t)=\theta_{1}\left(x, y, z_{1}, t\right), \\
& \alpha(x, y, z, t)=\alpha_{1}\left(x, y, z_{1}, t\right), \\
& v(x, y, z, t)=v_{1}\left(x, y, z_{1}, t\right), \\
& p(x, y, z, t)=p_{1}\left(x, y, z_{1}, t\right) .
\end{aligned}
$$

The system of (8)-(11) can be written in the form (index 1 for the new function is omitted)

$$
\begin{aligned}
\frac{\partial \theta}{\partial t}- & \frac{\partial \theta}{\partial z_{1}} \frac{\partial \zeta}{\partial t}+(v \cdot \widetilde{\nabla}) \theta \\
= & \widetilde{\Delta} \theta+Z \exp \left(\frac{\theta}{Z^{-1}+\delta \theta}\right) \phi(\alpha) \\
\frac{\partial \alpha}{\partial t}- & \frac{\partial \alpha}{\partial z_{1}} \frac{\partial \zeta}{\partial t}+(v \cdot \widetilde{\nabla}) \alpha=Z \exp \left(\frac{\theta}{Z^{-1}+\delta \theta}\right) \phi(\alpha) \\
\frac{\partial v}{\partial t}- & \frac{\partial v}{\partial z_{1}} \frac{\partial \zeta}{\partial t}+(v \cdot \tilde{\nabla}) v \\
= & -\widetilde{\nabla} p+P \widetilde{\Delta} v \\
& +Q\left(1+\lambda \sin _{1}\left(\sigma_{1} t\right)+\lambda_{2} \sin \left(\sigma_{2} t\right)\right)\left(\theta+\theta_{0}\right) \gamma \\
\frac{\partial v_{x}}{\partial x}- & \frac{\partial v_{x}}{\partial z_{1}} \frac{\partial \zeta}{\partial x}+\frac{\partial v_{y}}{\partial y}-\frac{\partial v_{y}}{\partial z_{1}} \frac{\partial \zeta}{\partial y}+\frac{\partial v_{z}}{\partial z_{1}}=0
\end{aligned}
$$

$$
\begin{aligned}
\widetilde{\Delta}= & \frac{\partial^{2}}{\partial x^{2}}+\frac{\partial^{2}}{\partial y^{2}}+\frac{\partial^{2}}{\partial z_{1}^{2}}-2 \frac{\partial^{2}}{\partial x \partial z_{1}} \frac{\partial \zeta}{\partial x}-2 \frac{\partial^{2}}{\partial y \partial z_{1}} \frac{\partial \zeta}{\partial y} \\
& +\frac{\partial^{2}}{\partial z_{1}^{2}}\left(\left(\frac{\partial \zeta}{\partial x}\right)^{2}+\left(\frac{\partial \zeta}{\partial y}\right)^{2}\right) \\
& -\frac{\partial}{\partial z_{1}}\left(\frac{\partial^{2} \zeta}{\partial x^{2}}+\frac{\partial^{2} \zeta}{\partial y^{2}}\right), \\
\widetilde{\nabla}= & \left(\frac{\partial}{\partial x}-\frac{\partial}{\partial z_{1}} \frac{\partial \zeta}{\partial x}, \frac{\partial}{\partial y}-\frac{\partial}{\partial z_{1}} \frac{\partial \zeta}{\partial y}, \frac{\partial}{\partial z_{1}}\right), \\
Q= & P R .
\end{aligned}
$$

To approximate the jump conditions and resolve the interface problem, we use the matched asymptotic expansion by seeking the outer solution of problem (14) in the form

$$
\begin{aligned}
& \theta=\theta_{0}+\epsilon \theta_{1}+\cdots, \\
& \alpha=\alpha_{0}+\epsilon \alpha_{1}+\cdots, \\
& v=v_{0}+\epsilon v_{1}+\cdots, \\
& p=p_{0}+\epsilon p_{1}+\cdots .
\end{aligned}
$$

Introducing the stretched coordinate $\eta=z_{1} \epsilon^{-1}$ where $\epsilon=$ $Z^{-1}$, the inner solution, can be approximated in the following form:

$$
\begin{aligned}
& \theta=\epsilon \widetilde{\theta}_{1}+\cdots, \\
& \alpha=\widetilde{\alpha}_{0}+\epsilon \widetilde{\alpha}_{1}+\cdots, \\
& v=\widetilde{v}_{0}+\epsilon \widetilde{v}_{1}+\cdots, \\
& p=\widetilde{p}_{0}+\epsilon \tilde{p}_{1}+\cdots, \\
& \zeta=\zeta_{0}+\epsilon \zeta_{1}+\cdots .
\end{aligned}
$$

Substituting the inner and outer solutions in (14) leads to $\operatorname{Order} \epsilon^{-2}$ :

$$
P\left(1+\left(\frac{\partial \zeta_{0}}{\partial x}\right)^{2}+\left(\frac{\partial \zeta_{0}}{\partial y}\right)^{2}\right) \frac{\partial^{2} \widetilde{v}_{0}}{\partial \eta^{2}}=0
$$

Order $\epsilon^{-1}$ :

$$
\begin{aligned}
& \left(1+\left(\frac{\partial \zeta_{0}}{\partial x}\right)^{2}+\left(\frac{\partial \zeta_{0}}{\partial y}\right)^{2}\right) \frac{\partial^{2} \widetilde{\theta}_{1}}{\partial \eta^{2}} \\
& +\exp \left(\frac{\widetilde{\theta}_{1}}{1+\delta \widetilde{\theta}_{1}}\right) \phi\left(\widetilde{\alpha}_{0}\right)=0 \\
& -\frac{\partial \widetilde{\alpha}_{0}}{\partial \eta} \frac{\partial \zeta_{0}}{\partial t}-\frac{\partial \widetilde{\alpha}_{0}}{\partial \eta}\left(\widetilde{v}_{0 x} \frac{\partial \zeta_{0}}{\partial x}+\widetilde{v}_{0 y} \frac{\partial \zeta_{0}}{\partial y}-\widetilde{v}_{0 z}\right) \\
& =\exp \left(\frac{\widetilde{\theta}_{1}}{1+\delta \widetilde{\theta}_{1}}\right) \phi\left(\widetilde{\alpha}_{0}\right), \\
& -\frac{\partial \widetilde{v}_{0 x}}{\partial \eta} \frac{\partial \zeta_{0}}{\partial t}-\widetilde{v}_{0 x} \frac{\partial \widetilde{v}_{0}}{\partial \eta} \frac{\partial \zeta_{0}}{\partial x}-\widetilde{v}_{0 y} \frac{\partial \widetilde{v}_{0}}{\partial \eta} \frac{\partial \zeta_{0}}{\partial y}+\widetilde{v}_{0 z} \frac{\partial \widetilde{v}_{0}}{\partial \eta} \\
& =t_{0} \frac{\partial \widetilde{p}_{0}}{\partial \eta}+P\left(A \frac{\partial^{2} \widetilde{v}_{1}}{\partial \eta^{2}}+F_{0} \frac{\partial \widetilde{v}_{0}}{\partial \eta}\right) \\
& -\frac{\partial \widetilde{v}_{0 x}}{\partial \eta} \frac{\partial \zeta_{0}}{\partial x}-\frac{\partial \widetilde{v}_{0 y}}{\partial \eta} \frac{\partial \zeta_{0}}{\partial y}+\frac{\partial \widetilde{v}_{0 z}}{\partial \eta}=0 .
\end{aligned}
$$


$\operatorname{Order} \epsilon^{0}:$

$$
\begin{gathered}
\frac{\partial \widetilde{v}_{0}}{\partial t}-\frac{\partial \widetilde{v}_{1}}{\partial \eta} \frac{\partial \zeta_{0}}{\partial t}-\frac{\partial \widetilde{v}_{0}}{\partial \eta} \frac{\partial \zeta_{1}}{\partial t}+\widetilde{v}_{0 x}\left(\frac{\partial \widetilde{v}_{0}}{\partial x}-\frac{\partial \widetilde{v}_{1}}{\partial \eta} \frac{\partial \zeta_{0}}{\partial x}\right. \\
\left.-\frac{\partial \widetilde{v}_{0}}{\partial \eta} \frac{\partial \zeta_{1}}{\partial x}\right)+\widetilde{v}_{1 x} \frac{\partial \widetilde{v}_{0}}{\partial \eta} \frac{\partial \zeta_{0}}{\partial x}+\widetilde{v}_{1 y} \frac{\partial \widetilde{v}_{0}}{\partial \eta} \frac{\partial \zeta_{0}}{\partial y} \\
+\widetilde{v}_{0 y}\left(\frac{\partial \widetilde{v}_{0}}{\partial y}-\frac{\partial \widetilde{v}_{1}}{\partial \eta} \frac{\partial \zeta_{0}}{\partial y}-\frac{\partial \widetilde{v}_{0}}{\partial \eta} \frac{\partial \zeta_{1}}{\partial y}\right)+\widetilde{v}_{0 z} \frac{\partial \widetilde{v}_{1}}{\partial \eta}+\widetilde{v}_{1 z} \\
. \frac{\partial \widetilde{v}_{0}}{\partial \eta}=-\nabla_{0} \widetilde{p}_{0}+t_{1} \frac{\partial \widetilde{p}_{0}}{\partial \eta}+t_{0} \frac{\partial \widetilde{p}_{1}}{\partial \eta}+P\left(A \frac{\partial^{2} \widetilde{v}_{2}}{\partial \eta^{2}}\right. \\
\left.+t_{3} \frac{\partial^{2} \widetilde{v}_{1}}{\partial \eta^{2}}+F_{0} \frac{\partial \widetilde{v}_{1}}{\partial \eta}+t_{4} \frac{\partial^{2} \widetilde{v}_{0}}{\partial \eta^{2}}+F_{1} \frac{\partial \widetilde{v}_{0}}{\partial \eta}+\Delta_{1} \widetilde{v}_{0}\right) \\
+Q\left(1+\lambda_{1} \sin \left(\sigma_{1} t\right)+\lambda_{2} \sin \left(\sigma_{2} t\right)\right) \gamma \theta_{0} \\
\frac{\partial \widetilde{v}_{0 x}}{\partial x}-\frac{\partial \widetilde{v}_{1 x}}{\partial \eta} \frac{\partial \zeta_{0}}{\partial x}-\frac{\partial \widetilde{v}_{0 x}}{\partial \eta} \frac{\partial \zeta_{1}}{\partial x}+\frac{\partial \widetilde{v}_{0 y}}{\partial y}-\frac{\partial \widetilde{v}_{1 y}}{\partial \eta} \frac{\partial \zeta_{0}}{\partial y} \\
-\frac{\partial \widetilde{v}_{0 y}}{\partial \eta} \frac{\partial \zeta_{1}}{\partial y}+\frac{\partial \widetilde{v}_{1 z}}{\partial \eta}=0 .
\end{gathered}
$$

$\operatorname{Order} \epsilon^{1}$ :

$$
\begin{aligned}
& \frac{\partial \widetilde{v}_{1}}{\partial t}-\left(\frac{\partial \widetilde{v}_{2}}{\partial \eta} \frac{\partial \zeta_{0}}{\partial t}+\frac{\partial \widetilde{v}_{1}}{\partial \eta} \frac{\partial \zeta_{1}}{\partial t}+\frac{\partial \widetilde{v}_{0}}{\partial \eta} \frac{\partial \zeta_{2}}{\partial t}\right)+\widetilde{v}_{0 x}\left(\frac{\partial \widetilde{v}_{1}}{\partial x}\right. \\
& \left.-\frac{\partial \widetilde{v}_{1}}{\partial \eta} \frac{\partial \zeta_{1}}{\partial x}-\frac{\partial \widetilde{v}_{0}}{\partial \eta} \frac{\partial \zeta_{2}}{\partial x}-\frac{\partial \widetilde{v}_{2}}{\partial \eta} \frac{\partial \zeta_{0}}{\partial x}\right)+\widetilde{v}_{1 x}\left(\frac{\partial \widetilde{v}_{0}}{\partial x}\right. \\
& \left.-\frac{\partial \widetilde{v}_{0}}{\partial \eta} \frac{\partial \zeta_{1}}{\partial x}-\frac{\partial \widetilde{v}_{1}}{\partial \eta} \frac{\partial \zeta_{0}}{\partial x}\right)-\widetilde{v}_{2 x} \frac{\partial \widetilde{v}_{0}}{\partial \eta} \frac{\partial \zeta_{0}}{\partial x}+\widetilde{v}_{0 y}\left(\frac{\partial \widetilde{v}_{1}}{\partial y}\right. \\
& \left.-\frac{\partial \widetilde{v}_{1}}{\partial \eta} \frac{\partial \zeta_{1}}{\partial y}-\frac{\partial \widetilde{v}_{0}}{\partial \eta} \frac{\partial \zeta_{2}}{\partial y}-\frac{\partial \widetilde{v}_{2}}{\partial \eta} \frac{\partial \zeta_{0}}{\partial y}\right)+\widetilde{v}_{1 y}\left(\frac{\partial \widetilde{v}_{0}}{\partial y}\right. \\
& \left.-\frac{\partial \widetilde{v}_{0}}{\partial \eta} \frac{\partial \zeta_{1}}{\partial y}-\frac{\partial \widetilde{v}_{1}}{\partial \eta} \frac{\partial \zeta_{0}}{\partial y}\right)-\widetilde{v}_{2 y} \frac{\partial \widetilde{v}_{0}}{\partial \eta} \frac{\partial \zeta_{0}}{\partial y}+\widetilde{v}_{0 z} \frac{\partial \widetilde{v}_{2}}{\partial \eta} \\
& +\widetilde{v}_{1 z} \frac{\partial \widetilde{v}_{1}}{\partial \eta}+\widetilde{v}_{2 z} \frac{\partial \widetilde{v}_{0}}{\partial \eta}=t_{0} \frac{\partial \widetilde{p}_{2}}{\partial \eta}+t_{1} \frac{\partial \widetilde{p}_{1}}{\partial \eta}-\nabla_{0} \widetilde{p}_{1}+t_{2} \\
& \quad+\frac{\partial \widetilde{p}_{0}}{\partial \eta}+P\left(A \frac{\partial^{2} \widetilde{v}_{3}}{\partial \eta^{2}}+t_{3} \frac{\partial^{2} \widetilde{v}_{2}}{\partial \eta^{2}}+F_{0} \frac{\partial \widetilde{v}_{2}}{\partial \eta}+F_{1} \frac{\partial \widetilde{v}_{1}}{\partial \eta}\right. \\
& \left.+\Delta_{1} \widetilde{v}_{1}+F_{2} \frac{\partial \widetilde{v}_{0}}{\partial \eta}\right)+Q\left(1+\lambda_{1} \sin \left(\sigma_{1} t\right)\right. \\
& \left.+\lambda_{2} \sin \left(\sigma_{2} t\right)\right) \theta_{0} \gamma,
\end{aligned}
$$$$
\frac{\partial \widetilde{v}_{1 x}}{\partial x}-\frac{\partial \widetilde{v}_{2 x}}{\partial \eta} \frac{\partial \zeta_{0}}{\partial x}-\frac{\partial \widetilde{v}_{1 x}}{\partial \eta} \frac{\partial \zeta_{1}}{\partial x}-\frac{\partial \widetilde{v}_{0 x}}{\partial \eta} \frac{\partial \zeta_{2}}{\partial x}+\frac{\partial \widetilde{v}_{1 y}}{\partial y}
$$$$
-\frac{\partial \widetilde{v}_{2 y}}{\partial \eta} \frac{\partial \zeta_{0}}{\partial y}-\frac{\partial \widetilde{v}_{1 y}}{\partial \eta} \frac{\partial \zeta_{1}}{\partial y}-\frac{\partial \widetilde{v}_{0 y}}{\partial \eta} \frac{\partial \zeta_{2}}{\partial y}+\frac{\partial \widetilde{v}_{2 z}}{\partial \eta}=0 .
$$

Order $\epsilon^{2}$ :

$$
\begin{aligned}
\frac{\partial \widetilde{v}_{2 x}}{\partial x}- & \frac{\partial \widetilde{v}_{3 x}}{\partial \eta} \frac{\partial \zeta_{0}}{\partial x}-\frac{\partial \widetilde{v}_{2 x}}{\partial \eta} \frac{\partial \zeta_{1}}{\partial x}-\frac{\partial \widetilde{v}_{1 x}}{\partial \eta} \frac{\partial \zeta_{2}}{\partial x}-\frac{\partial \widetilde{v}_{0 x}}{\partial \eta} \frac{\partial \zeta_{3}}{\partial x} \\
+ & \frac{\partial \widetilde{v}_{2 y}}{\partial y}-\frac{\partial \widetilde{v}_{3 y}}{\partial \eta} \frac{\partial \zeta_{0}}{\partial y}-\frac{\partial \widetilde{v}_{2 y}}{\partial \eta} \frac{\partial \zeta_{1}}{\partial y}-\frac{\partial \widetilde{v}_{1 y}}{\partial \eta} \frac{\partial \zeta_{2}}{\partial y} \\
& -\frac{\partial \widetilde{v}_{0 y}}{\partial \eta} \frac{\partial \zeta_{3}}{\partial y}+\frac{\partial \widetilde{v}_{3 z}}{\partial \eta}=0,
\end{aligned}
$$

where

$$
\begin{aligned}
\Delta_{1} & =\frac{\partial^{2}}{\partial x^{2}}+\frac{\partial^{2}}{\partial y^{2}}, \\
\nabla_{0} & =\left(\frac{\partial}{\partial x}, \frac{\partial}{\partial y}, 0\right), \\
F_{i} & =-2\left(\frac{\partial \zeta_{i}}{\partial x} \frac{\partial}{\partial x}+\frac{\partial \zeta_{i}}{\partial y} \frac{\partial}{\partial y}\right)-\left(\frac{\partial^{2} \zeta_{i}}{\partial x^{2}}+\frac{\partial^{2} \zeta_{i}}{\partial y^{2}}\right) I \\
t_{0} & =\left(\frac{\partial \zeta_{0}}{\partial x}, \frac{\partial \zeta_{0}}{\partial y},-1\right), \quad i=0, \\
t_{i} & =\left(\frac{\partial \zeta_{i}}{\partial x}, \frac{\partial \zeta_{i}}{\partial y},-1\right), i=1,2, \\
t_{3} & =2\left(\frac{\partial \zeta_{1}}{\partial x} \frac{\partial \zeta_{0}}{\partial x}+\frac{\partial \zeta_{0}}{\partial y} \frac{\partial \zeta_{1}}{\partial y}\right), \\
t_{4} & =\left(\frac{\partial \zeta_{1}}{\partial x}\right)^{2}+\left(\frac{\partial \zeta_{1}}{\partial y}\right)^{2}+\frac{\partial \zeta_{0}}{\partial x} \frac{\partial \zeta_{2}}{\partial x}+\frac{\partial \zeta_{0}}{\partial y} \frac{\partial \zeta_{2}}{\partial y}, \\
A & =\left(1+\left(\frac{\partial \zeta_{0}}{\partial x}\right)^{2}+\left(\frac{\partial \zeta_{0}}{\partial y}\right)^{2}\right)
\end{aligned}
$$$$
i=0,1,2 \text {, }
$$

and $I$ is the identity operator. The matching conditions as $\eta \rightarrow \pm \infty$ are given by

$$
\begin{aligned}
\widetilde{v}_{0} & \left.\sim v_{0}\right|_{z_{1}= \pm 0}, \\
\widetilde{v}_{1} & \sim\left(\left.\frac{\partial v_{0}}{\partial z_{1}}\right|_{z_{1}= \pm 0}\right) \eta+\left.v_{1}\right|_{z_{1}= \pm 0}, \\
\widetilde{v}_{2} & \sim \frac{1}{2}\left(\left.\frac{\partial^{2} v_{0}}{\partial z_{1}^{2}}\right|_{z_{1}= \pm 0}\right) \eta^{2}+\left(\left.\frac{\partial v_{1}}{\partial z_{1}}\right|_{z_{1}= \pm 0}\right) \eta \\
& +\left.v_{2}\right|_{z_{1}= \pm 0}, \\
\widetilde{v}_{3} & \sim \frac{1}{6}\left(\left.\frac{\partial^{3} v_{0}}{\partial z_{1}^{3}}\right|_{z_{1}= \pm 0}\right) \eta^{3}+\frac{1}{2}\left(\left.\frac{\partial^{2} v_{1}}{\partial z_{1}^{2}}\right|_{z_{1}= \pm 0}\right) \eta^{2} \\
& +\left(\left.\frac{\partial v_{2}}{\partial z_{1}}\right|_{z_{1}= \pm 0}\right) \eta+\left.v_{3}\right|_{z_{1}= \pm 0} .
\end{aligned}
$$


As $\eta \rightarrow+\infty$

$$
\left.\tilde{\theta}_{1} \approx \theta_{1}\right|_{z_{1}=+0}+\left(\left.\frac{\partial \theta_{0}}{\partial z_{1}}\right|_{z_{1}=+0}\right) \eta, \quad \widetilde{\alpha}_{0} \longrightarrow 0 .
$$

As $\eta \rightarrow-\infty$

$$
\left.\tilde{\theta}_{1} \sim \theta_{1}\right|_{z_{1}=-0}, \quad \tilde{\alpha}_{0} \longrightarrow 1
$$

From (18), we obtain

$$
\frac{\partial^{2} \widetilde{v}_{0}}{\partial \eta^{2}}=0
$$

Consequently, we can conclude that $\widetilde{v}_{0}(\eta)$ is a linear function of $\eta$ and identically constant since the velocity is bounded. Equation (29) becomes

$$
\begin{aligned}
\left.v_{0}\right|_{z_{1}=+0} & =\left.v_{0}\right|_{z_{1}=-0}, \\
\frac{\partial \widetilde{v}_{0}}{\partial \eta} & =0 .
\end{aligned}
$$

From (36)-(37), we deduce that the first term in the expression of the velocity $v_{0}$ is continuous at the front. By substituting (37) into (21), we find

$$
\begin{array}{r}
P A \frac{\partial^{2} \widetilde{v}_{1}}{\partial \eta^{2}}+t_{0} \frac{\partial \widetilde{p}_{0}}{\partial \eta}=0 \\
\frac{\partial \widetilde{v}_{0 x}}{\partial x}-\frac{\partial \widetilde{v}_{1 x}}{\partial \eta} \frac{\partial \zeta_{0}}{\partial x}+\frac{\partial \widetilde{v}_{0 y}}{\partial y}-\frac{\partial \widetilde{v}_{1 y}}{\partial \eta} \frac{\partial \zeta_{0}}{\partial y}+\frac{\partial \widetilde{v}_{1 z}}{\partial \eta}=0 .
\end{array}
$$

Differentiating (39) with respect to $\eta$, one obtains

$$
\frac{\partial^{2} \widetilde{v}_{1 x}}{\partial \eta^{2}} \frac{\partial \zeta_{0}}{\partial x}+\frac{\partial^{2} \widetilde{v}_{1 y}}{\partial \eta^{2}} \frac{\partial \zeta_{0}}{\partial y}-\frac{\partial^{2} \widetilde{v}_{1 z}}{\partial \eta^{2}}=0
$$

As a result, (38) is a vectorial equation with three components. We multiply the first component by $\partial \zeta_{0} / \partial x$, the second by $\partial \zeta_{0} / \partial y$, and the third by -1 and adding we have

$$
P A\left(\frac{\partial^{2} \widetilde{v}_{1 x}}{\partial \eta^{2}} \frac{\partial \zeta_{0}}{\partial x}+\frac{\partial^{2} \widetilde{v}_{1 y}}{\partial \eta^{2}} \frac{\partial \zeta_{0}}{\partial y}-\frac{\partial^{2} \widetilde{v}_{1 z}}{\partial \eta^{2}}\right)+A \frac{\partial \widetilde{p}_{0}}{\partial \eta}=0 .
$$

Considering the two equations (38) and (40), we obtain

$$
\begin{gathered}
\frac{\partial \widetilde{p}_{0}}{\partial \eta}=0 \\
\frac{\partial^{2} \widetilde{v}_{1}}{\partial \eta^{2}}=0 .
\end{gathered}
$$

From the boundedness of the velocity and (30), the continuity of the first order term of the outer expansion and of the first derivative of the zero-order term, we can write

$$
\begin{gathered}
\left.\frac{\partial v_{0}}{\partial z_{1}}\right|_{z_{1}=+0}=\left.\frac{\partial v_{0}}{\partial z_{1}}\right|_{z_{1}=-0}, \\
\left.v_{1}\right|_{z_{1}=+0}=\left.v_{1}\right|_{z_{1}=-0} .
\end{gathered}
$$

Differentiating (23) once and (26) twice with respect to $\eta$ and using the three equations (37), (42), and (43), we get

$$
t_{0} \frac{\partial^{2} \widetilde{p}_{1}}{\partial \eta^{2}}+P A \frac{\partial^{3} \widetilde{v}_{2}}{\partial \eta^{3}}=0
$$

$$
\frac{\partial^{3} \widetilde{v}_{2 x}}{\partial \eta^{3}} \frac{\partial \zeta_{0}}{\partial x}+\frac{\partial^{3} \widetilde{v}_{2 y}}{\partial \eta^{3}} \frac{\partial \zeta_{0}}{\partial y}-\frac{\partial^{3} \widetilde{v}_{2 z}}{\partial \eta^{3}}=0 .
$$

As above, we multiply the three components of (45), respectively, by $\partial \zeta_{0} / \partial x, \partial \zeta_{0} / \partial y$, and -1 and adding, we get

$$
P A\left(\frac{\partial^{3} \widetilde{v}_{2 x}}{\partial \eta^{3}} \frac{\partial \zeta_{0}}{\partial x}+\frac{\partial^{3} \widetilde{v}_{2 y}}{\partial \eta^{3}} \frac{\partial \zeta_{0}}{\partial y}-\frac{\partial^{3} \widetilde{v}_{2 z}}{\partial \eta^{3}}\right)+A \frac{\partial^{2} \widetilde{p}_{1}}{\partial \eta^{2}}=0 .
$$

From the previous equation and using (45)-(46), we have

$$
\begin{aligned}
& \frac{\partial^{2} \widetilde{p}_{1}}{\partial \eta^{2}}=0 \\
& \frac{\partial^{3} \widetilde{v}_{2}}{\partial \eta^{3}}=0 .
\end{aligned}
$$

Knowing that the velocity is bounded and considering (31), we obtain the jump conditions in the following forms:

$$
\begin{gathered}
\left.\frac{\partial^{2} v_{0}}{\partial z_{1}^{2}}\right|_{z_{1}=+0}=\left.\frac{\partial^{2} v_{0}}{\partial z_{1}^{2}}\right|_{z_{1}=-0}, \\
\left.\frac{\partial v_{1}}{\partial z_{1}}\right|_{z_{1}=+0}=\left.\frac{\partial v_{1}}{\partial z_{1}}\right|_{z_{1}=-0}, \\
\left.v_{2}\right|_{z_{1}=+0}=\left.v_{2}\right|_{z_{1}=-0} .
\end{gathered}
$$

Differentiating (25) twice and (27) three times with respect to $\eta$ and taking into account (37), (42), (48), we obtain

$$
\begin{aligned}
& t_{0} \frac{\partial^{3} \widetilde{p}_{2}}{\partial \eta^{3}}+P A \frac{\partial^{4} \widetilde{v}_{3}}{\partial \eta^{4}} \\
& +Q\left(1+\lambda_{1} \sin \left(\sigma_{1} t\right)+\lambda_{2} \sin \left(\sigma_{2} t\right)\right) \gamma \frac{\partial^{2} \widetilde{\theta}_{1}}{\partial \eta^{2}}=0 \\
& \frac{\partial^{4} \widetilde{v}_{3 x}}{\partial \eta^{4}} \frac{\partial \zeta_{0}}{\partial x}+\frac{\partial^{4} \widetilde{v}_{3 y}}{\partial \eta^{4}} \frac{\partial \zeta_{0}}{\partial y}-\frac{\partial^{4} \widetilde{v}_{3 z}}{\partial \eta^{4}}=0 \\
& P A\left(\frac{\partial^{4} \widetilde{v}_{3 x}}{\partial \eta^{4}} \frac{\partial \zeta_{0}}{\partial x}+\frac{\partial^{4} \widetilde{v}_{3 y}}{\partial \eta^{4}} \frac{\partial \zeta_{0}}{\partial y}-\frac{\partial^{4} \widetilde{v}_{3 z}}{\partial \eta^{4}}\right)+A \frac{\partial^{3} \widetilde{p}_{2}}{\partial \eta^{3}} \\
& \quad-Q\left(1+\lambda_{1} \sin \left(\sigma_{1} t\right)+\lambda_{2} \sin \left(\sigma_{2} t\right)\right) \frac{\partial^{2} \widetilde{\theta}_{1}}{\partial \eta^{2}}=0
\end{aligned}
$$

From the last equation and using (50), we can write

$$
\begin{aligned}
& A \frac{\partial^{3} \tilde{p}_{2}}{\partial \eta^{3}}-Q\left(1+\lambda_{1} \sin \left(\sigma_{1} t\right)+\lambda_{2} \sin \left(\sigma_{2} t\right)\right) \frac{\partial^{2} \widetilde{\theta}_{1}}{\partial \eta^{2}} \\
& \quad=0 \\
& \gamma_{0} \frac{\partial^{2} \widetilde{\theta}_{1}}{\partial \eta^{2}}=\frac{\partial^{4} \widetilde{v}_{3}}{\partial \eta^{4}}
\end{aligned}
$$


where $\gamma_{0}$ is defined by

$$
\begin{aligned}
\gamma_{0}= & \left(-\frac{\partial \zeta_{0}}{\partial x} \frac{R}{A^{2}},-\frac{\partial \zeta_{0}}{\partial y} \frac{R}{A^{2}}, \frac{R}{A^{2}}-\frac{R}{A}\right) \\
& \cdot\left(1+\lambda_{1} \sin \left(\sigma_{1} t\right)+\lambda_{2} \sin \left(\sigma_{2} t\right)\right) .
\end{aligned}
$$

Now, integrating (53) with respect to $\eta$ using the system (32)-(34) lead to

$$
\left.\frac{\partial^{3} v_{0}}{\partial z_{1}^{3}}\right|_{z_{1}=-0}-\left.\frac{\partial^{3} v_{0}}{\partial z_{1}^{3}}\right|_{z_{1}=+0}=-\left.\gamma_{0} \frac{\partial \theta_{0}}{\partial z_{1}}\right|_{z_{1}=+0}
$$

As a first conclusion, the velocity jump conditions across the reaction front are given by (36), (44), (49), and (55).

From (20), (22), (37), we conclude that $\widetilde{\alpha}_{0}$ is a monotonic function satisfying $0<\widetilde{\alpha}_{0}<1$. Therefore, the reaction is of zero order and $\phi\left(\widetilde{\alpha}_{0}\right) \equiv 1$. Multiplying (19) by $\partial \widetilde{\theta}_{1} / \partial \eta$ and integrating the result yield

$$
\begin{aligned}
& \left.\left(\frac{\partial \widetilde{\theta}_{1}}{\partial \eta}\right)^{2}\right|_{+\infty}-\left.\left(\frac{\partial \widetilde{\theta}_{1}}{\partial \eta}\right)^{2}\right|_{-\infty} \\
& =2 A^{-1} \int_{-\infty}^{\theta_{1} \mid z_{1}=-0} \exp \left(\frac{\tau}{1+\tau \delta}\right) d \tau .
\end{aligned}
$$
obtains

Subtracting (19) from (20) and integrating the result one

$$
\left.\frac{\partial \widetilde{\theta}_{1}}{\partial \eta}\right|_{+\infty}-\left.\frac{\partial \widetilde{\theta}_{1}}{\partial \eta}\right|_{-\infty}=-A^{-1}\left(\frac{\partial \zeta_{0}}{\partial t}+s\right),
$$

where $s$ is given by

$$
s=\widetilde{v}_{0 x} \frac{\partial \zeta_{0}}{\partial x}+\widetilde{v}_{0 y} \frac{\partial \zeta_{0}}{\partial y}-\widetilde{v}_{0 z} .
$$

As a second conclusion, the temperature jump conditions across the reaction front are given by the two last equations (56)-(57). By using the matching conditions above and truncating the expansion as

$$
\begin{aligned}
\theta & \approx \theta_{0}, \\
\left.\theta_{1}\right|_{z_{1}=-0} & \left.\approx Z \theta\right|_{z_{1}=+0}, \\
\zeta & \approx \zeta_{0}, \\
v & \approx v_{0},
\end{aligned}
$$

the jump conditions can be written as

$$
\begin{aligned}
& \left.\left(\frac{\partial \theta}{\partial z_{1}}\right)^{2}\right|_{+0}-\left.\left(\frac{\partial \theta}{\partial z_{1}}\right)^{2}\right|_{-0} \\
& =2 Z\left(1+\left(\frac{\partial \zeta}{\partial x}\right)^{2}+\left(\frac{\partial \zeta}{\partial y}\right)^{2}\right)^{-1} \\
& \cdot \int_{-\infty}^{\left.\theta_{1}\right|_{z_{1}=-0}} \exp \left(\frac{\tau}{Z^{-1}+\tau \delta}\right) d \tau \\
& \left.\frac{\partial \theta}{\partial z_{1}}\right|_{z_{1}=+0}-\left.\frac{\partial \theta}{\partial z_{1}}\right|_{z_{1}=-0} \\
& =-\left(1+\left(\frac{\partial \zeta}{\partial x}\right)^{2}+\left(\frac{\partial \zeta}{\partial y}\right)^{2}\right)^{-1} \\
& \cdot\left(\frac{\partial \zeta}{\partial t}+\left.\left(v_{x} \frac{\partial \zeta}{\partial x}+v_{y} \frac{\partial \zeta}{\partial y}-v_{z}\right)\right|_{z_{1}=+0}\right) \\
& \left.v_{z}\right|_{z_{1}=+0}=\left.v_{z}\right|_{z_{1}=-0} \text {, } \\
& \left.\frac{\partial v_{z}}{\partial z_{1}}\right|_{z_{1}=+0}=\left.\frac{\partial v_{z}}{\partial z_{1}}\right|_{z_{1}=-0}, \\
& \left.\frac{\partial^{2} v_{z}}{\partial z_{1}^{2}}\right|_{z_{1}=+0}=\left.\frac{\partial v_{z}}{\partial z_{1}^{2}}\right|_{z_{1}=-0}, \\
& \left.\frac{\partial^{3} v_{z}}{\partial z_{1}^{3}}\right|_{z_{1}=-0}-\left.\frac{\partial^{3} v_{z}}{\partial z_{1}^{3}}\right|_{z_{1}=+0} \\
& =-R\left(1+\left(\frac{\partial \zeta}{\partial x}\right)^{2}+\left(\frac{\partial \zeta}{\partial y}\right)^{2}\right)^{-1} \\
& \cdot\left(\left(1+\left(\frac{\partial \zeta}{\partial x}\right)^{2}+\left(\frac{\partial \zeta}{\partial y}\right)^{2}\right)^{-1}-1\right) \\
& \left.\cdot\left(1+\lambda_{1} \sin \left(\sigma_{1} t\right)+\lambda_{2} \sin \left(\sigma_{2} t\right)\right) \frac{\partial \theta}{\partial z_{1}}\right|_{z_{1}=+0} .
\end{aligned}
$$

\section{The Interface Problem and Perturbation}

To study the propagation of polymerization front with a liquid reactant and liquid product, the equation of motion has been considered before and after the reaction zone. This changes the jump conditions and influences the stability conditions of the frontal polymerization process. Notice that in the case of liquid-solid polymerization front, the equation of motion is considered after the reaction zone. The original system (2), (3), (8), (9) leads to the following interface problem:

In the liquid monomer $z>\zeta$

$$
\begin{aligned}
& \frac{\partial \theta}{\partial t}+(v \cdot \nabla) \theta=\Delta \theta, \\
& \alpha=0
\end{aligned}
$$




$$
\begin{aligned}
\frac{\partial v}{\partial t}+ & (v \cdot \nabla) v \\
= & -\nabla p+P \Delta v \\
& +Q\left(1+\lambda_{1} \sin \sigma_{1} t+\lambda_{2} \sin \sigma_{2} t\right)\left(\theta+\theta_{0}\right) \gamma, \\
\operatorname{div}(v) & =0 .
\end{aligned}
$$

In the liquid polymer $z<\zeta$

$$
\begin{aligned}
& \frac{\partial \theta}{\partial t}+(v \cdot \nabla) \theta=\Delta \theta \\
& \alpha=1 \text {, } \\
& \frac{\partial v}{\partial t}+(v \cdot \nabla) v \\
& =-\nabla p+P \Delta v \\
& +Q\left(1+\lambda_{1} \sin \sigma_{1} t+\lambda_{2} \sin \sigma_{2} t\right)\left(\theta+\theta_{0}\right) \gamma
\end{aligned}
$$

$\operatorname{div}(v)=0$.

$$
\text { At the interface } z=\zeta
$$$$
\left.\theta\right|_{\zeta-0}=\left.\theta\right|_{\zeta+0},
$$$$
\left.\frac{\partial \theta}{\partial z}\right|_{\zeta=-0}-\left.\frac{\partial \theta}{\partial z}\right|_{\zeta=+0}=\left(1+\left(\frac{\partial \zeta}{\partial x}\right)^{2}+\left(\frac{\partial \zeta}{\partial y}\right)^{2}\right)^{-1}
$$$$
\cdot\left(\frac{\partial \zeta}{\partial t}+\left.\left(v_{x} \frac{\partial \zeta}{\partial x}+v_{y} \frac{\partial \zeta}{\partial y}-v_{z}\right)\right|_{\zeta}\right)
$$$$
\left.\left(\frac{\partial \theta}{\partial z}\right)^{2}\right|_{\zeta-0}-\left.\left(\frac{\partial \theta}{\partial z}\right)^{2}\right|_{\zeta_{1}+0}
$$$$
=-2 Z\left(1+\left(\frac{\partial \zeta}{\partial x}\right)^{2}+\left(\frac{\partial \zeta}{\partial y}\right)^{2}\right)^{-1}
$$$$
\cdot \int_{-\infty}^{\left.\theta\right|_{\zeta}} \exp \left(\frac{\tau}{Z^{-1}+\delta \tau}\right) d \tau
$$$$
\left.v_{z}\right|_{\zeta=-0}=\left.v_{z}\right|_{\zeta=+0},
$$$$
\left.\frac{\partial v_{z}}{\partial z}\right|_{\zeta=-0}=\left.\frac{\partial v_{z}}{\partial z}\right|_{\zeta=+0},
$$$$
\left.\frac{\partial^{2} v_{z}}{\partial z^{2}}\right|_{\zeta=-0}=\left.\frac{\partial^{2} v_{z}}{\partial z^{2}}\right|_{\zeta=+0}
$$$$
\left.\frac{\partial^{3} v_{z}}{\partial z^{3}}\right|_{\zeta=-0}-\left.\frac{\partial^{3} v_{z}}{\partial z^{3}}\right|_{\zeta=+0}
$$

$$
\begin{aligned}
& =-R\left(1+\left(\frac{\partial \zeta}{\partial x}\right)^{2}+\left(\frac{\partial \zeta}{\partial y}\right)^{2}\right)^{-1} \\
& \cdot\left(\left(1+\left(\frac{\partial \zeta}{\partial x}\right)^{2}+\left(\frac{\partial \zeta}{\partial y}\right)^{2}\right)^{-1}-1\right) \\
& \left.\cdot\left(1+\lambda_{1} \sin \left(\sigma_{1} t\right)+\lambda_{2} \sin \left(\sigma_{2} t\right)\right)\left(\frac{\partial \theta}{\partial z}\right)\right|_{\zeta=+0},
\end{aligned}
$$

with the conditions at infinity

$$
\begin{aligned}
& z=-\infty: \quad \theta=0, v=0, \\
& z=+\infty: \quad \theta=-1, \quad v=0 .
\end{aligned}
$$

\section{Stability Analysis}

The interface problem analyzed in Section 4 has a travelling wave solution in the following form:

$$
\begin{aligned}
& (\theta(x, y, z, t), \alpha(x, y, z, t), v) \\
& \quad=\left(\theta_{s}(z-u t), \alpha_{s}(z-u t), 0\right), \\
& \left(\theta_{s}(z-u t), \alpha_{s}(z-u t)\right) \\
& \quad= \begin{cases}(0,1), & z_{2}<0 \\
\left(\exp \left(-u z_{2}\right)-1,0\right), & z_{2}>0,\end{cases}
\end{aligned}
$$

where $z_{2}=z-u t$ and $u$ is the speed of the stationary reaction front. This solution is a basic and stationary solution of interface problem (61)-(70) written in the moving coordinates. Then, (61), (63), (65), and (67) can be replaced by

$$
\begin{aligned}
\frac{\partial \theta}{\partial t}+ & (v \cdot \nabla) \theta=\Delta \theta+u \frac{\partial \theta}{\partial z_{2}}, \\
\frac{\partial v}{\partial t}+ & (v \nabla) v \\
= & -\nabla p+P \Delta v+u \frac{\partial \theta}{\partial z_{2}} \\
& +Q\left(1+\lambda_{1} \sin \left(\sigma_{1} t\right)+\lambda_{2} \sin \left(\sigma_{2} t\right)\right)\left(\theta+\theta_{0}\right) \gamma .
\end{aligned}
$$

The other equations remain unchanged. To study the stability of reaction front, we seek the solution of the problem as follows:

$$
\begin{aligned}
& \theta=\theta_{s}+\tilde{\theta}, \\
& p=p_{s}+\tilde{p}, \\
& v=v_{s}+\widetilde{v},
\end{aligned}
$$

where $\widetilde{\theta}, \widetilde{p}$, and $\widetilde{v}$ are, respectively, small perturbation of temperature, pressure, and velocity. 
Subtituting (73) into (64), (72), one obtains the first-order terms as follows:

In the liquid monomer $z_{2}>\xi$

$$
\begin{aligned}
\frac{\partial \widetilde{\theta}}{\partial t}= & \Delta \widetilde{\theta}+u \frac{\partial \widetilde{\theta}}{\partial z_{2}}-\widetilde{v}_{z} \theta_{s}^{\prime}, \\
\frac{\partial \widetilde{v}}{\partial t}= & -\nabla \widetilde{p}+P \Delta \widetilde{v}+u \frac{\partial \widetilde{\theta}}{\partial z_{2}} \\
& +Q\left(1+\lambda_{1} \sin \left(\sigma_{1} t\right)+\lambda_{2} \sin \left(\sigma_{2} t\right)\right) \tilde{\theta} \gamma
\end{aligned}
$$$$
\operatorname{div}(\widetilde{v})=0 .
$$

In the liquid polymer $z_{2}<\xi$

$$
\begin{aligned}
\frac{\partial \tilde{\theta}}{\partial t}= & \Delta \widetilde{\theta}+u \frac{\partial \widetilde{\theta}}{\partial z_{2}}, \\
\frac{\partial \widetilde{v}}{\partial t}= & -\nabla \widetilde{p}+P \Delta \widetilde{v}+u \frac{\partial \widetilde{v}}{\partial z_{2}} \\
& +Q\left(1+\lambda_{1} \sin \left(\sigma_{1} t\right)+\lambda_{2} \sin \left(\sigma_{2} t\right)\right) \tilde{\theta} \gamma
\end{aligned}
$$

$\operatorname{div}(\widetilde{v})=0$,

where $\xi$ is defined by $\xi=\zeta-u t$.

We note

$$
\left(\widetilde{\theta}, \widetilde{v}_{z}\right)= \begin{cases}\left(\widehat{\theta}_{1}, \widehat{v}_{z 1}\right) & \text { for } z_{2}<\xi \\ \left(\widehat{\theta}_{2}, \widehat{v}_{z 2}\right) & \text { for } z_{2}<\xi\end{cases}
$$

and consider the perturbation as follows:

$$
\begin{aligned}
\widehat{\theta}_{i} & =\theta_{i}\left(z_{2}, t\right) \exp \left(j\left(k_{1} x+k_{2} y\right)\right), \\
\widehat{v}_{z i} & =v_{z i}\left(z_{2}, t\right) \exp \left(j\left(k_{1} x+k_{2} y\right)\right), \\
\xi & =\epsilon_{1}(t) \exp \left(j\left(k_{1} x+k_{2} y\right)\right),
\end{aligned}
$$

where $k_{i}, i=1,2$, and $\epsilon_{1}$ denote, respectively, the wave numbers (in $x$ and $y$ directions) and the amplitude of the perturbation and $j^{2}=-1$. Then, jump conditions (69) are linearized by taking into account the fact that

$$
\begin{aligned}
\left.\theta\right|_{\xi= \pm 0} & =\theta_{s}( \pm 0)+\xi \theta_{s}^{\prime}( \pm 0)+\widetilde{\theta}( \pm 0), \\
\left.\frac{\partial \theta}{\partial z_{2}}\right|_{\xi= \pm 0} & =\theta_{s}^{\prime}( \pm 0)+\xi \theta_{s}^{\prime \prime}( \pm 0)+\left.\frac{\partial \widetilde{\theta}}{\partial z_{2}}\right|_{\xi= \pm 0} .
\end{aligned}
$$

Then the higher-order terms are given as

$$
\begin{gathered}
\left.\widehat{\theta}_{2}\right|_{z_{2}=0}-\left.\widehat{\theta}_{1}\right|_{z_{2}=0}=u \xi, \\
\left.\frac{\partial \widehat{\theta}_{2}}{\partial z_{2}}\right|_{z_{2}=0}-\left.\frac{\partial \widehat{\theta}_{1}}{\partial z_{2}}\right|_{z_{2}=0}=-u^{2} \xi-\frac{\partial \xi}{\partial t}+\left.\widetilde{v}_{z}\right|_{z_{2}=0},
\end{gathered}
$$

$$
u^{2} \xi+\left.\frac{\partial \widehat{\theta}_{2}}{\partial z_{2}}\right|_{z_{2}=0}=-\left.\frac{Z}{u} \widehat{\theta}_{1}\right|_{z_{2}=0},
$$

$$
\left.\widehat{v}_{2 z}\right|_{z_{2}=0}=\left.\widehat{v}_{1 z}\right|_{z_{2}=0},
$$

$$
\left.\frac{\partial \widehat{v}_{z_{2}}}{\partial z_{2}}\right|_{z_{2}=0}=\left.\frac{\partial \widehat{v}_{1 z}}{\partial z_{2}}\right|_{z_{2}=0},
$$

$$
\left.\frac{\partial^{2} \widehat{v}_{2 z}}{\partial z_{2}^{2}}\right|_{z_{2}=0}=\left.\frac{\partial^{2} \widehat{v}_{1 z}}{\partial z_{2}^{2}}\right|_{z_{2}=0} \text {, }
$$

$$
\left.\frac{\partial^{3} \widehat{v}_{2 z}}{\partial z_{2}^{3}}\right|_{z_{2}=0}=\left.\frac{\partial^{3} \widehat{v}_{1 z}}{\partial z_{2}^{3}}\right|_{z_{2}=0} \text {. }
$$

Substituting (81) into (83)-(89) leads to

$$
\begin{aligned}
\theta_{2}(0, t)-\theta_{1}(0, t) & =u \epsilon_{1}(t), \\
\theta_{2}^{\prime}(0, t)-\theta_{1}^{\prime}(0, t) & =-\epsilon_{1}(t) u^{2}-\epsilon_{1}^{\prime}(t)+v_{1}(0, t), \\
\epsilon_{1}(t) u^{2}+\theta_{2}^{\prime}(0, t) & =-\frac{Z}{u} \theta_{1}(0, t), \\
v_{2}^{(i)}(0, t) & =v_{1}^{(i)}(0, t) \quad i=0,1,2,3,
\end{aligned}
$$

where

$$
\begin{aligned}
v^{(i)} & =\frac{\partial^{i} v}{\partial v^{i}}, \\
\theta^{\prime} & =\frac{\partial \theta_{i}}{\partial z}, \\
\epsilon_{1}^{\prime}(t) & =\frac{d \epsilon_{1}(t)}{d t} .
\end{aligned}
$$

After simplification, (75) and (78) become

$$
\begin{aligned}
& \frac{\partial}{\partial t} \Delta \widetilde{v}_{z}-u \frac{\partial}{\partial z_{2}} \Delta \widetilde{v}_{z}=P \Delta \Delta \widetilde{v}_{z}+Q\left(1+\lambda_{1} \sin \left(\sigma_{1} t\right)\right. \\
& \left.+\lambda_{2} \sin \left(\sigma_{2} t\right)\left(\frac{\partial^{2}}{\partial x^{2}}+\frac{\partial^{2}}{\partial y^{2}}\right)\right) \widetilde{\theta}
\end{aligned}
$$

Substituting (71), (87), (88) into (74), (77), (92) and introducing the variable $w=v^{\prime \prime}-k^{2} v$, where $k=\sqrt{k_{1}^{2}+k_{2}^{2}}$, one obtains two systems of equations as follows:

$$
\begin{aligned}
& z_{2}<\xi \\
& \begin{aligned}
\frac{\partial w}{\partial t} & -u w^{\prime}-P\left(w^{\prime \prime}-k^{2} w\right) \\
& =-Q k^{2}\left(1+\lambda_{1} \sin \left(\sigma_{1} t\right)+\lambda_{2} \sin \left(\sigma_{2} t\right)\right) \theta, \\
w & =v^{\prime \prime}-k^{2} v, \\
\frac{\partial \theta}{\partial t} & -\theta^{\prime \prime}-u \theta^{\prime}+k^{2} \theta=0 .
\end{aligned}
\end{aligned}
$$




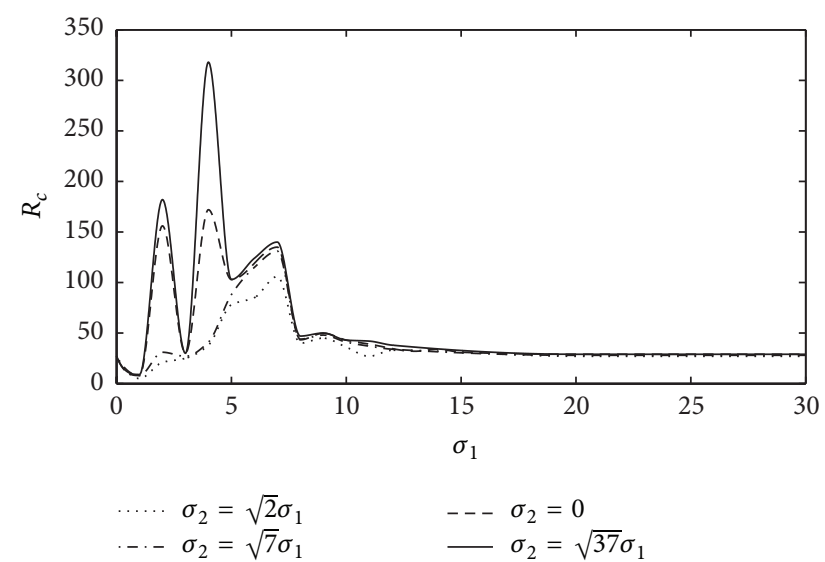

FIGURE 1: Critical Rayleigh number as a function of $\sigma_{1}$ for different frequencies ratio and for $\sigma_{2}=0, \lambda_{1}=\lambda_{2}=5, P=0.5, Z=8$, and $k=0.7$.

$$
\begin{aligned}
& z_{2}>\xi: \\
& \frac{\partial w}{\partial t}-u w^{\prime}-P\left(w^{\prime \prime}-k^{2} w\right) \\
& \quad=-Q k^{2}\left(1+\lambda_{1} \sin \left(\sigma_{1} t\right)+\lambda_{2} \sin \left(\sigma_{2} t\right)\right) \theta, \\
& \begin{aligned}
w & =v^{\prime \prime}-k^{2} v, \\
\frac{\partial \theta}{\partial t} & -\theta^{\prime \prime}-u \theta^{\prime}+k^{2} \theta=u \exp \left(-u z_{2}\right) v,
\end{aligned}
\end{aligned}
$$

with the following boundary conditions:

$$
\begin{aligned}
\theta_{2}(0, t)-\theta_{1}(0, t) & =u \epsilon_{1}(t), \\
\theta_{2}^{\prime}(0, t)-\theta_{1}^{\prime}(0, t) & =-u^{2} \varepsilon_{1}(t)-\varepsilon_{1}^{\prime}(t)+v_{1}(0, t), \\
\theta_{2}^{\prime}(0, t)+\frac{Z}{u} \theta_{1}(0, t) & =-u^{2} \varepsilon_{1}(t), \\
v_{1}^{(i)}(0, t) & =v_{2}^{(i)}(0, t), \quad i=0,1,2,3 .
\end{aligned}
$$
yields

Combining the three boundary conditions (95)-(97)

$$
\begin{aligned}
\theta_{1}^{\prime}(0, t)+\frac{Z}{u} \theta_{1}(0, t)= & \frac{1}{u}\left(\theta_{2}(0, t)-\theta_{1}(0, t)\right)_{t}^{\prime} \\
& -v_{1}(0, t)
\end{aligned}
$$

\section{Numerical Results}

The numerical simulations of the problem are performed using the finite-difference approximation with implicit scheme. The algorithm is given in Appendix.

Evolution of maximum temperature versus time provides the onset of stability of the polymerization front. The convective instability occurs when a jump from bounded to unbounded values of maximum temperature is achieved.

Figure 1 shows the critical Rayleigh number as a function of the frequency $\sigma_{1}$ for different frequencies ratio and for

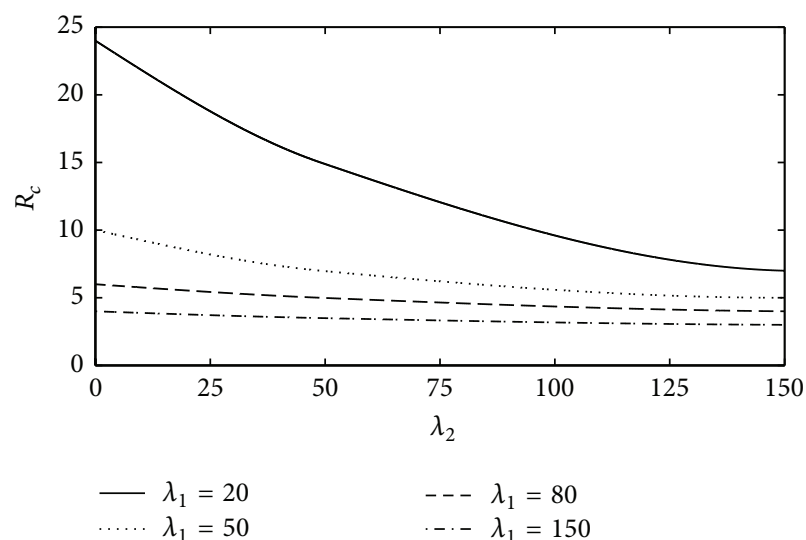

FIGURE 2: Critical Rayleigh number as a function of $\lambda_{2}$ for different values of $\lambda_{1}, \sigma_{2}=\sqrt{2} \sigma_{1}, \sigma_{1}=5, P=0.5, Z=8$, and $k=0.7$.

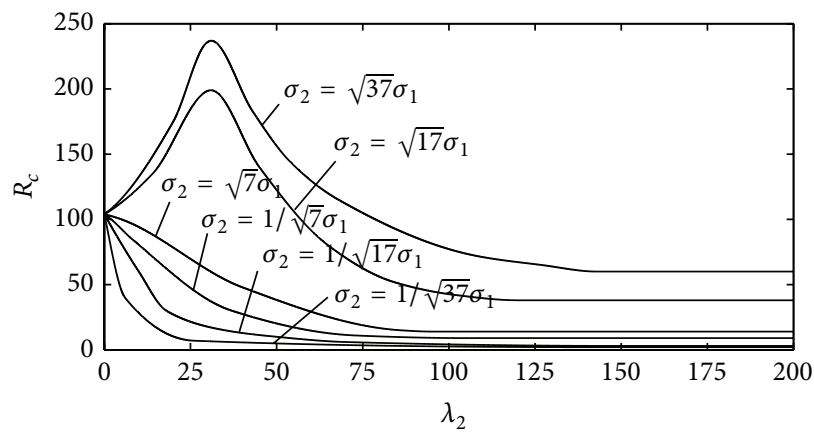

FIgURE 3: Critical Rayleigh number as a function of $\lambda_{2}$ for different frequencies ratio, $k=0.7, \lambda_{1}=5, P=0.5, Z=8$, and $\sigma_{1}=5$.

$\sigma_{2}=0$. In the absence of gravitational modulation $\left(\sigma_{1}=\sigma_{2}=\right.$ $0)$, we find the same result $\left(R_{c} \approx 27\right)$ as in $[8,14]$. It can be observed that the frequencies ratio of the $\mathrm{QP}$ gravitational modulation has a significant influence on the convective instability of the reaction front. As indicated, increasing the frequencies ratio, the stability domain becomes larger. In particular, this can be seen in the range of the frequency $\sigma_{1}$ belonging to the interval $(0,10)$. For large values of the frequency $\sigma_{1}$, the frequencies ratio $\sigma_{2} / \sigma_{1}$ has no effect on the convective instability. It is also worthy to notice that the QP gravitational modulation has a stabilizing effect for sufficiently large frequencies ratio $\left(\sigma_{2}=\sqrt{37} \sigma_{1}\right)$ comparing to the periodic modulation case $\left(\sigma_{2}=0\right)$.

The critical Rayleigh number as a function of the amplitude $\lambda_{2}$ is shown in Figure 2 for different values of $\lambda_{1}$. We can conclude that, for a fixed value of $\lambda_{2}$ and increased values of $\lambda_{1}$, the polymerization front loses its stability monotonically revealing that the stability of the reaction front can be controlled by acting on the amplitudes of the QP modulation.

Figure 3 illustrates the critical Rayleigh number as a function of the amplitude $\lambda_{2}$ for different frequencies ratio. It can be observed that increasing the frequencies ratio the stability domain becomes large for certain values of $\lambda_{2}$. For large values of $\lambda_{2}\left(\lambda_{2} \geq 100\right)$, the effect of the frequencies ratio on the convective instability boundary is insignificant. 


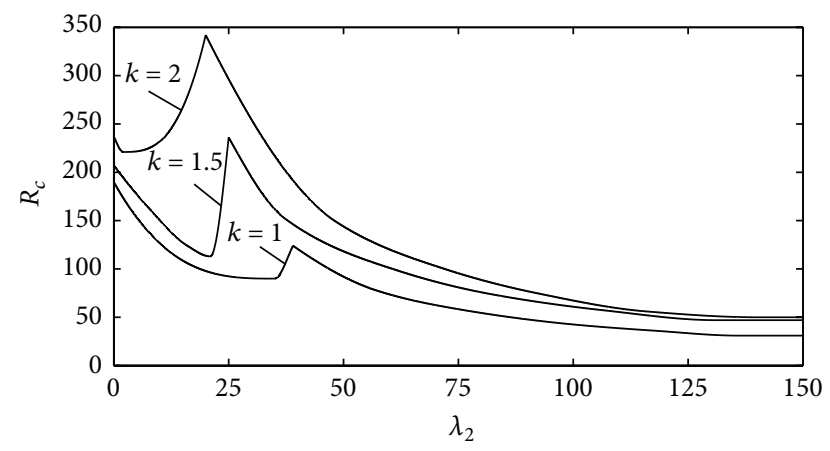

Figure 4: Critical Rayleigh number as a function of $\lambda_{2}$ for different values of number wave, $\sigma_{2}=\sqrt{7} \sigma_{1}, \sigma_{1}=10, P=0.5, Z=8$, and $\lambda_{1}=5$.

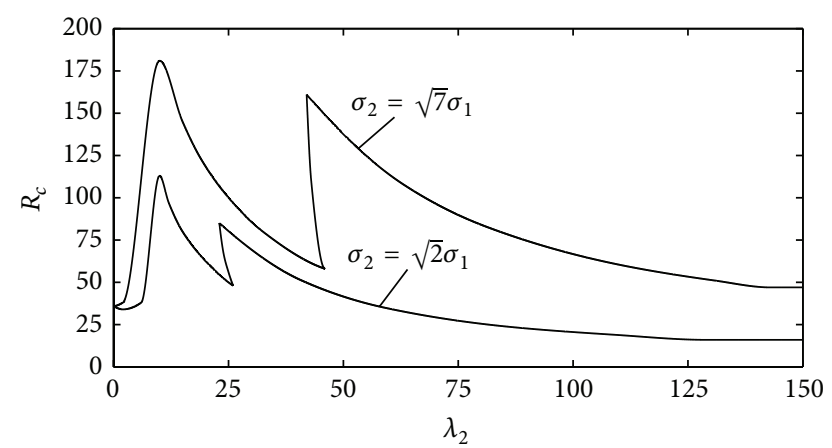

FIgURE 5: Critical Rayleigh number as a function of $\lambda_{2}$ for two different frequencies ratios, $k=0.7, \lambda_{1}=5, P=0.5, Z=8$, and $\sigma_{1}=10$.

Figure 4 shows for a fixed frequencies ratio, $\sigma_{2} / \sigma_{1}=\sqrt{7}$, the influence of the wave number on the critical Rayleigh number versus $\lambda_{2}$. The plots indicate that for large values of the wave number the reaction front gains stability.

The influence of $\lambda_{2}$ on the stability of the reaction front is given in Figure 5 for two different frequencies ratio. From this figure, one can conclude that an increase of the frequencies ratio has a stabilizing effect.

\section{Conclusion}

In this work, we have studied the influence of the QP vibrations on the convective instability of liquid-liquid polymerization front. We have used the model which includes the heat equation, the concentration equation, and the Navier-Stokes equations under Boussinesq approximation. The Zeldovich Frank-Kamenetskii method has been used assuming that the reaction occurs in a narrow zone. To obtain the convective instability threshold using the linear stability analysis, the reduced system of equations has been discretized and resolved using the finite-difference method with implicit scheme. The results have shown that, for fixed values of amplitudes, an increase of the frequencies ratio stabilizes the reaction front, and for large values of the frequency $\sigma_{1}$ the critical Rayleigh number tends to the unmodulated critical value. In addition, it is observed that for a fixed frequencies ratio and for a given amplitude $\lambda_{2}$ an increase of the amplitude $\lambda_{1}$ destabilizes the reaction front. Also, the reaction front becomes more stable by increasing the wave number.

\section{Appendix}

The algorithm of the considered problem uses the finitedifference approximation with implicit scheme. The velocity is computed from the previous time values of the problem. The discretization in space is given by $z_{i}=i h$, where $h$ is the space step and $i$ is a positive integer. The discretization in time is given by $t_{i}=i \tau$, where $\tau$ is sufficiently small time step. The variables of discretization are $w_{i}^{j}=w\left(z_{i}, t_{j}\right), v_{i}^{j}=v\left(z_{i}, t_{j}\right)$, and $\theta_{i}^{j}=\theta\left(z_{i}, t_{j}\right)$. One obtains the following:

In the liquid monomer $z<\zeta$

$$
\begin{aligned}
& \left(\frac{-1}{h^{2}}\right) \theta_{i-1}^{j+1}+\left(\frac{1}{\tau}+\frac{2}{h^{2}}+k^{2}+\frac{u}{h}\right) \theta_{i}^{j+1} \\
& \quad+\left(-\frac{1}{h^{2}}-\frac{u}{h}\right) \theta_{i+1}^{j+1}=\frac{1}{\tau} \theta_{i}^{j}, \\
& \left(\frac{-P}{h^{2}}\right) w_{i-1}^{j+1}+\left(\frac{1}{\tau}+\frac{2 P}{h^{2}}+P k^{2}+\frac{u}{h}\right) w_{i}^{j+1} \\
& \quad+\left(-\frac{P}{h^{2}}-\frac{u}{h}\right) w_{i+1}^{j+1}=\frac{1}{\tau} w_{i}^{j} \\
& \quad-Q k^{2}\left(1+\lambda_{1} \sin \left(\sigma_{1} t_{j}\right)+\lambda_{2} \sin \left(\sigma_{2} t_{j}\right)\right) \theta_{i}^{j}, \\
& \left(\frac{1}{h^{2}}\right) v_{i-1}^{j+1}+\left(-\frac{2}{h^{2}}-k^{2}\right) v_{i}^{j+1}+\left(\frac{1}{h^{2}}\right) v_{i+1}^{j+1}=w_{i}^{j} .
\end{aligned}
$$

In the liquid polymer $z>\zeta$

$$
\begin{aligned}
& \left(\frac{-1}{h^{2}}\right) T_{i-1}^{j+1}+\left(\frac{1}{\tau}+\frac{2}{h^{2}}+k^{2}+\frac{u}{h}\right) T_{i}^{j+1} \\
& \quad+\left(-\frac{1}{h^{2}}-\frac{u}{h}\right) T_{i+1}^{j+1}=\frac{1}{\tau} T_{i}^{j}+u \exp \left(-u z_{i}\right) V_{i}^{j}, \\
& \left(\frac{-P}{h^{2}}\right) W_{i-1}^{j+1}+\left(\frac{1}{\tau}+\frac{2 P}{h^{2}}+P k^{2}+\frac{u}{h}\right) W_{i}^{j+1} \\
& \quad+\left(-\frac{P}{h^{2}}-\frac{u}{h}\right) W_{i+1}^{j+1}=\frac{1}{\tau} W_{i}^{j} \\
& \quad-Q k^{2}\left(1+\lambda_{1} \sin \left(\sigma_{1} t_{j}\right)+\lambda_{2} \sin \left(\sigma_{2} t_{j}\right)\right) T_{i}^{j}, \\
& \left(\frac{1}{h^{2}}\right) V_{i-1}^{j+1}+\left(-\frac{2}{h^{2}}-k^{2}\right) V_{i}^{j+1}+\left(\frac{1}{h^{2}}\right) V_{i+1}^{j+1}=W_{i}^{j} .
\end{aligned}
$$

From the jump conditions, we have also the following systems:

$$
\begin{aligned}
& \left(\frac{-1}{h^{2}}\right) \theta_{1}^{j+1} \\
& \quad+\left(\frac{1}{\tau}+\frac{2}{h^{2}}+k^{2}+\frac{u}{h}+\left(\frac{c_{1}}{c_{2}}\right)\left(\frac{1}{h^{2}}+\frac{u}{h}\right)\right) \theta_{0}^{j+1}
\end{aligned}
$$




$$
\begin{aligned}
& =\left(\frac{-1}{\tau}\right) \theta_{0}^{j}-\left(\frac{1}{u \tau c_{2}}\right)\left(\frac{1}{h^{2}}+\frac{u}{h}\right)\left(T_{0}^{j}-\theta_{0}^{j}\right) \\
& -\left(\frac{v_{0}}{c_{2}}\right)\left(\frac{1}{h^{2}}+\frac{u}{h}\right) \\
& \left(\frac{-P}{h^{2}}\right) w_{1}^{j+1}+\left(\frac{1}{\tau}+\frac{2 P}{h^{2}}+P k^{2}+\frac{u}{h}\right) w_{0}^{j+1} \\
& +\left(\frac{-P}{h^{2}}-\frac{u}{h}\right) W_{0}^{j+1}=\left(\frac{-1}{\tau}\right) w_{1}^{j} \\
& +Q k^{2}\left(1+\lambda_{1} \sin \left(\sigma_{1} t_{j}\right)+\lambda_{2} \sin \left(\sigma_{2} t_{j}\right)\right) \\
& \cdot\left(\left(\frac{-c_{2}}{c_{1}}\right) T_{0}-\frac{1}{u \tau c_{1}}\left(T_{0}^{j}-\theta_{0}^{j}\right)-\frac{v_{0}}{c_{1}}\right) \text {, } \\
& \left(\frac{1}{\tau}+\frac{2}{h^{2}}+k^{2}+\frac{u}{h}+\frac{c_{2}}{h^{2} c_{1}}\right) T_{0}^{j+1}+\left(\frac{-1}{h^{2}}-\frac{u}{h}\right) T_{1}^{j+1} \\
& =\left(\frac{-1}{\tau}\right) T_{0}^{j}-u \exp (-u h)-\left(\frac{1}{u \tau h^{2} c_{1}}\right)\left(T_{0}^{j}-\theta_{0}^{j}\right) \\
& -\frac{v_{0}}{h^{2} c_{1}} \\
& \left(\frac{-P}{h^{2}}\right) w_{1}^{j+1}+\left(\frac{1}{\tau}+\frac{2 P}{h^{2}}+P k^{2}+\frac{u}{h}\right) w_{0}^{j+1} \\
& +\left(\frac{-P}{h^{2}}-\frac{u}{h}\right) W_{0}^{j+1}=\left(\frac{-1}{\tau}\right) w_{0}^{j} \\
& +Q k^{2}\left(1+\lambda_{1} \sin \left(\sigma_{1} t_{j}\right)+\lambda_{2} \sin \left(\sigma_{2} t_{j}\right)\right) \\
& \cdot\left(\left(\frac{-c_{1}}{c_{2}}\right) \theta_{0}-\frac{1}{u \tau c_{2}}\right)\left(T_{0}^{j}-\theta_{0}^{j}-\frac{v_{0}}{c_{2}}\right),
\end{aligned}
$$

with

$$
\begin{aligned}
& c_{1}=\left(\frac{1}{h}-\frac{Z}{u}-\frac{1}{u \tau}\right), \\
& c_{2}=\left(-\frac{1}{h}+\frac{1}{u \tau}\right) .
\end{aligned}
$$

\section{Conflict of Interests}

The authors declare that there is no conflict of interests regarding the publication of this paper.

\section{References}

[1] B. B. Khanukaev, M. A. Kozhushner, N. S. Enikolopyan, and N. M. Chechilo, "Theory of the propagation of a polymerization front," Combustion, Explosion, and Shock Waves, vol. 10, no. 1, pp. 17-21, 1974.

[2] J. A. Pojman and T.-C.-M. Qui, Nonlinear Dynamics with Polymers: Fundamentals, Methods and Applications, John Wiley \& Sons, 2011.

[3] I. D. Robertson, H. L. Hernandez, S. R. White, and J. S. Moore, "Rapid stiffening of a microfluidic endoskeleton via frontal polymerization," ACS Applied Materials and Interfaces, vol. 6, no. 21, pp. 18469-18474, 2014.

[4] Vi. Volpert and Vl. Volpert, "Propagation of frontal polymerization-crystallization waves," European Journal of Applied Mathematics, vol. 5, no. 2, pp. 201-215, 1994.

[5] G. Bowden, M. Garbey, V. M. Ilyashenko et al., "Effect of convection on a propagating front with a solid product: comparison of theory and experiments," The Journal of Physical Chemistry B, vol. 101, no. 4, pp. 678-686, 1997.

[6] M. Garbey, A. Taik, and V. Volpert, "Linear stability analysis of reaction fronts in liquids," Quarterly of Applied Mathematics, vol. 54, no. 2, pp. 225-247, 1996.

[7] K. Allali, V. Volpert, and J. A. Pojman, "Influence of vibrations on convective instability of polymerization fronts," Journal of Engineering Mathematics, vol. 41, no. 1, pp. 13-31, 2001.

[8] K. Allali, F. Bikany, A. Taik, and V. Volpert, "Influence of vibrations on convective instability of reaction fronts in liquids," Mathematical Modelling of Natural Phenomena, vol. 5, no. 7, pp. 35-41, 2010.

[9] K. Allali, M. Belhaq, and K. El Karouni, "Influence of quasiperiodic gravitational modulation on convective instability of reaction fronts in porous media," Communications in Nonlinear Science and Numerical Simulation, vol. 17, no. 4, pp. 1588-1596, 2012.

[10] K. Allali, S. Assiyad, and M. Belhaq, "Convection of polymerization front with solid product under quasi-periodic gravitational modulation," Nonlinear Dynamics and Systems Theory, vol. 14, no. 4, pp. 323-334, 2014.

[11] M. Menzinger and R. Wolfgang, "The meaning and use of the Arrhenius activation energy," Angewandte Chemie International Edition in English, vol. 8, no. 6, pp. 438-444, 1969.

[12] B. V. Novozhilov, "The rate of propagation of the front of an exothermic reaction in a condensed phase," Doklady Physical Chemistry, vol. 141, pp. 836-838, 1961.

[13] Y. B. Zeldovich and D. A. Frank-Kamenetskii, "A theory of thermal propagation of ame," Acta Physicochimica USSR, vol. 9, pp. 341-350, 1938.

[14] M. Garbey, A. Taïk, and V. Volpert, "Influence of natural convection on stability of reaction fronts in liquids," Quarterly of Applied Mathematics, vol. 56, no. 1, pp. 1-35, 1998. 


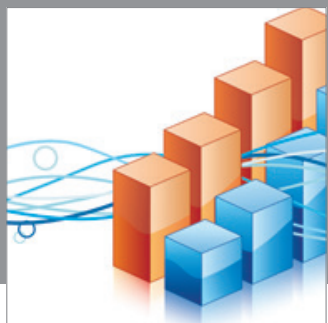

Advances in

Operations Research

mansans

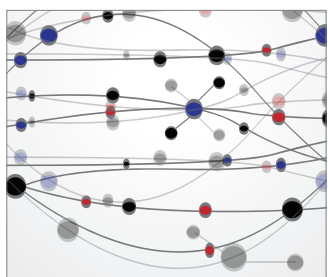

The Scientific World Journal
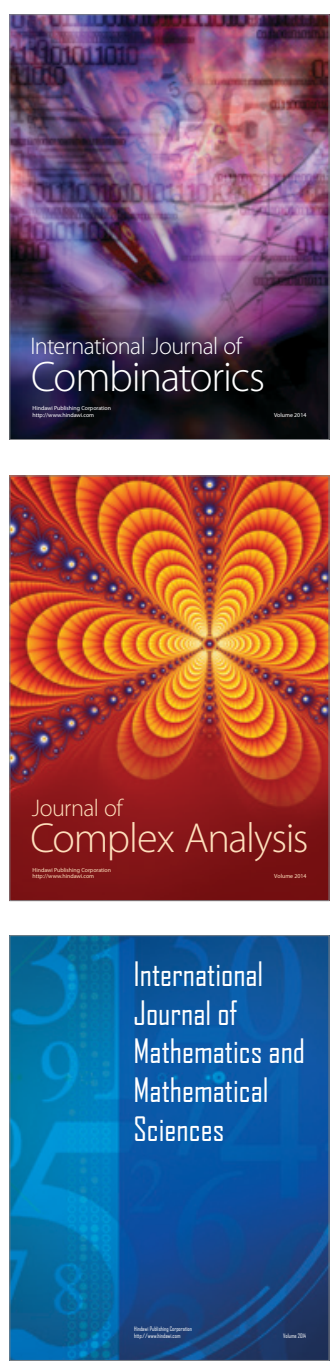
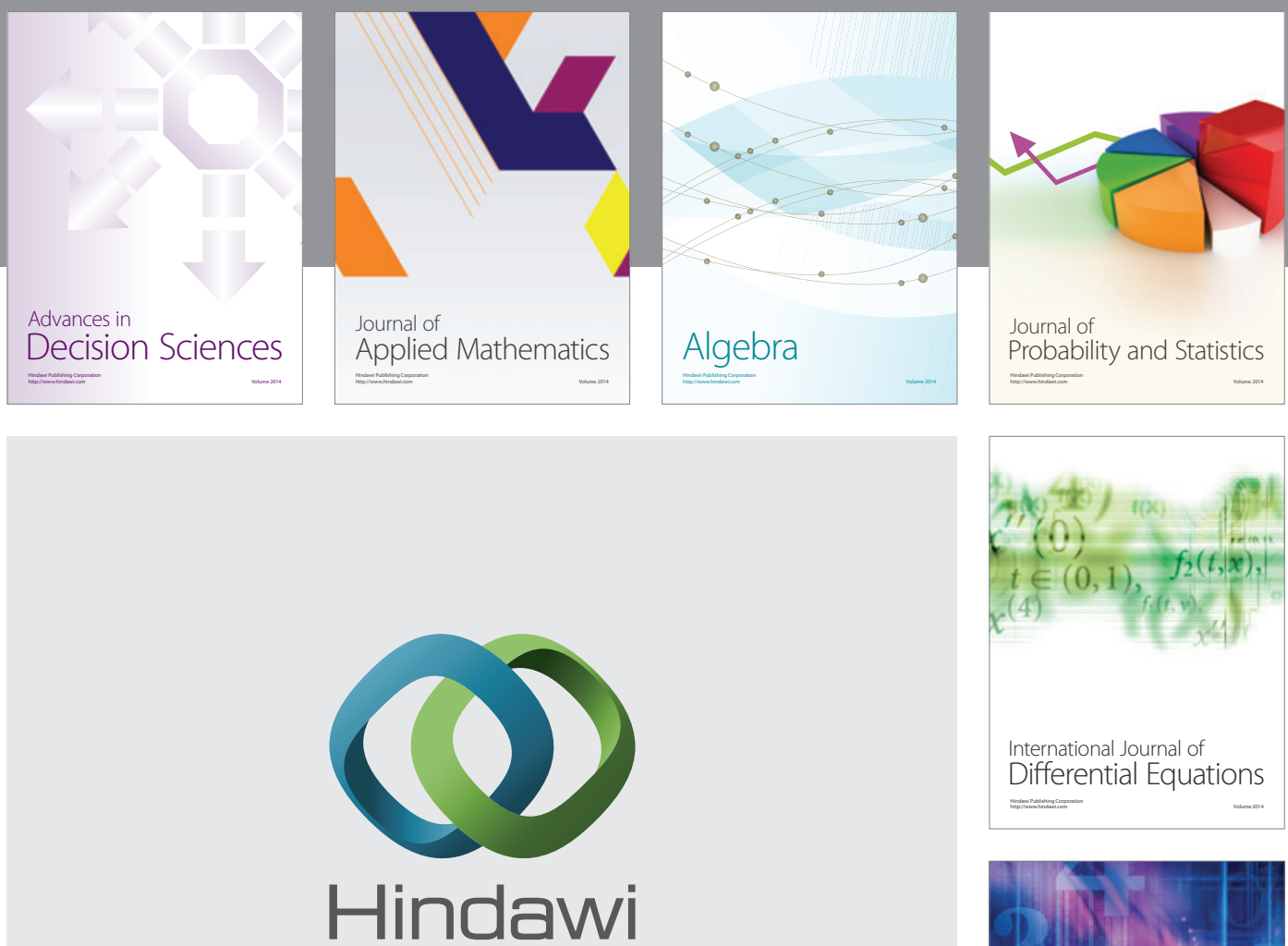

Submit your manuscripts at http://www.hindawi.com
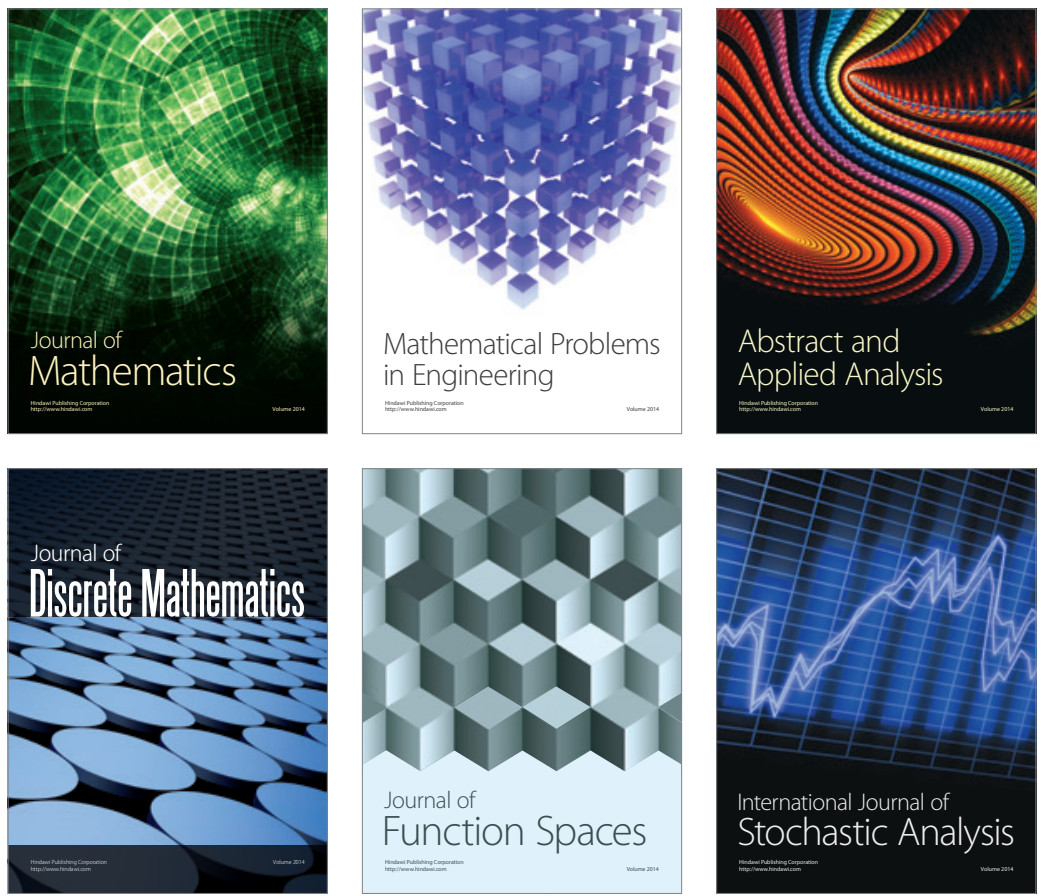

Journal of

Function Spaces

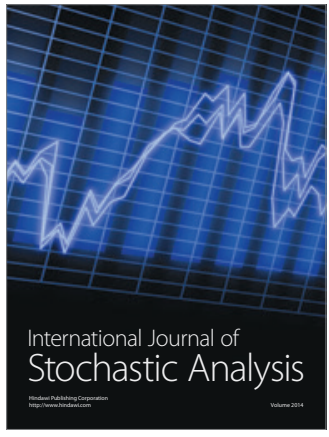

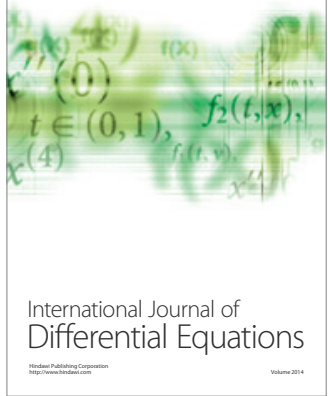
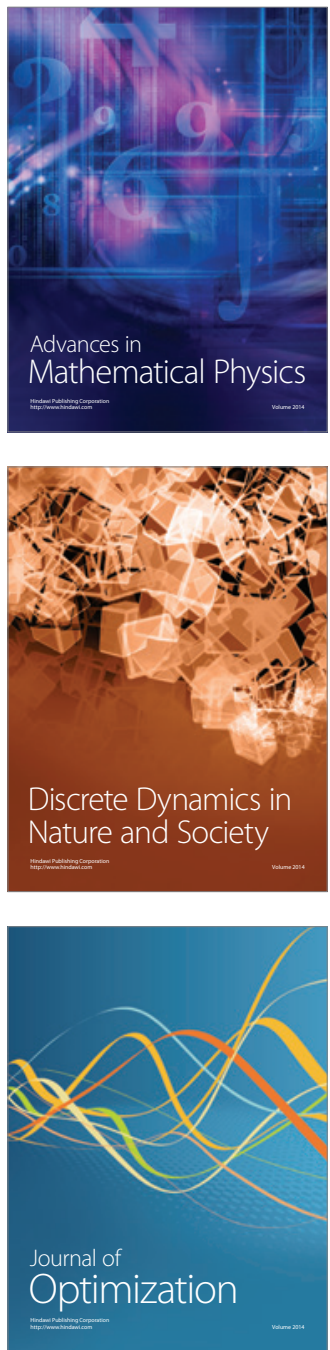